Nonlinear Processes in Geophysics, 12, 491-503, 2005

SRef-ID: $1607-7946 / \mathrm{npg} / 2005-12-491$

European Geosciences Union

(C) 2005 Author(s). This work is licensed

under a Creative Commons License.

\title{
Ensemble Kalman filter assimilation of temperature and altimeter data with bias correction and application to seasonal prediction
}

\author{
C. L. Keppenne ${ }^{1}$, M. M. Rienecker ${ }^{2}$, N. P. Kurkowski ${ }^{1}$, and D. A. Adamec ${ }^{3}$ \\ ${ }^{1}$ Science Applications International Corporation, 4600 Powder Mill Road, Beltsville, Maryland 20705, USA \\ ${ }^{2}$ Global Modeling and Assimilation Office, Mail Code 610.1, Goddard Space Flight Center, Greenbelt, Maryland 20771, USA \\ ${ }^{3}$ Oceans and Ice Branch, Laboratory for Hydrospheric Processes, Mail Code 614.2, Goddard Space Flight Center, Greenbelt, \\ Maryland 20771, USA
}

Received: 29 September 2004 - Revised: 7 February 2005 - Accepted: 18 April 2005 - Published: 17 May 2005

Part of Special Issue "Quantifying predictability"

\begin{abstract}
To compensate for a poorly known geoid, satellite altimeter data is usually analyzed in terms of anomalies from the time mean record. When such anomalies are assimilated into an ocean model, the bias between the climatologies of the model and data is problematic. An ensemble Kalman filter (EnKF) is modified to account for the presence of a forecast-model bias and applied to the assimilation of TOPEX/Poseidon (T/P) altimeter data. The online bias correction (OBC) algorithm uses the same ensemble of model state vectors to estimate biased-error and unbiased-error covariance matrices. Covariance localization is used but the bias covariances have different localization scales from the unbiased-error covariances, thereby accounting for the fact that the bias in a global ocean model could have much larger spatial scales than the random error.
\end{abstract}

The method is applied to a 27-layer version of the Poseidon global ocean general circulation model with about 30million state variables. Experiments in which T/P altimeter anomalies are assimilated show that the $\mathrm{OBC}$ reduces the RMS observation minus forecast difference for sea-surface height ( $\mathrm{SSH}$ ) over a similar EnKF run in which OBC is not used. Independent in situ temperature observations show that the temperature field is also improved. When the T/P data and in situ temperature data are assimilated in the same run and the configuration of the ensemble at the end of the run is used to initialize the ocean component of the GMAO coupled forecast model, seasonal SSH hindcasts made with the coupled model are generally better than those initialized with optimal interpolation of temperature observations without altimeter data. The analysis of the corresponding sea-surface temperature hindcasts is not as conclusive.

Correspondence to: C. L. Keppenne

(keppenne@gsfc.nasa.gov)

\section{Introduction}

Although sequential data assimilation algorithms rely on the assumption of an unbiased model, this assumption is almost always violated. The severity of the violation varies from model to model, but systematic patterns are common to many models. For example, many ocean general circulation models (OGCMs) underestimate the amplitude of the annual cycle of upper-ocean temperature in the tropics, resulting in a cold bias in the summer and a warm bias in the winter (in this paper, the terms bias and systematic errors are used interchangeably, while the term random error identifies the part of the forecast error that has zero expectation.).

Methods to deal with bias were introduced decades ago in the engineering community. In contrast, in the atmosphere and ocean modeling community, widespread interest in addressing systematic forecast model errors is more recent. This is understandable since bias only became a major concern after advances in modeling and data assimilation had reduced random errors to the point of commensurability with systematic errors.

Bias correction approaches can be separated into offline methods - in which the bias is estimated beforehand from the model and observed climatologies - and online methods - where a prior estimate of the bias is updated, resulting in an analyzed bias. Several recent online bias correction approaches use a two-stage estimation technique (Dee and da Silva, 1998; Dee and Todling, 2000; Carton et al., 2000; Martin et al., 2002; Chepurin et al., $2005^{1}$ ). Originally introduced by Friedland (1969) among signal processing engineers, the two-stage techniques augment the model state vector with an estimate of the bias. It is also assumed that

\footnotetext{
${ }^{1}$ Chepurin, G., Carton, J. A., and Dee, D. : Forecast model bias correction in ocean data assimilation. Mon. Wea. Rev., under review, 2005.
} 
the bias can be "uncoupled" from the other state-vector variables, thereby allowing the bias estimation and state estimation to occur successively.

To update the prior bias estimate, the two-stage estimation methods make assumptions about the biased-error and unbiased-error covariance distributions. In this regard, inspiration can be drawn from our knowledge of the backgrounderror distribution. Accordingly, Dee and da Silva (1998) followed by Dee and Todling (2000) and Martin et al. (2002) - assume that the two distributions are identical except for a proportionality factor. Another approach encountered in the recent literature proceeds as follows. In a conventional 3DVAR system, the error-covariance distributions are characterized by their amplitudes and horizontal and vertical decorrelation scales. Therefore, Carton et al. (2000) and Chepurin et al. (2005) $)^{1}$ estimate parameters of the bias and random-error covariance distributions offline by separating the basin-scale and synoptic-scale components of the observed-minus-forecast (OMF) differences. These works make clear that the performance of the assimilation system can be significantly enhanced when different covariance models are used for the systematic and unbiased errors.

How the prior estimate of the bias is assumed to change with time is another important component of a biasestimation method. A steady state model can be assumed in which $\boldsymbol{b}_{k}^{f}$, the prior bias estimate at time $t_{k}$, is identical to $\boldsymbol{b}_{k-1}^{a}$, the posterior bias estimate at the time, $t_{k-1}$, of the previous analysis (e.g. Dee and Todling, 2000; Martin et al., 2002). Alternatively, Chepurin et al. (2005) ${ }^{1}$ use regression analysis to fit simple bias models to the large-scale OMF differences. In their work, the prior bias estimate has a temporal component (i.e. $\boldsymbol{b}_{k}^{f}=\boldsymbol{F}\left(t_{k}\right)$ ) but the posterior bias estimate is not dynamically evolved (as in $\boldsymbol{b}_{k}^{f}=\boldsymbol{F}\left(\boldsymbol{b}_{k-1}^{a}, t_{k}\right)$ ).

This paper builds upon the aforementioned studies from the perspective of applying the ensemble Kalman filter (EnKF) - augmented with an online bias correction (OBC) algorithm - to assimilate SSH anomaly measurements from TOPEX/Poseidon (T/P) and in situ temperature data into a high-resolution global primitive-equation OGCM. The assimilation methodology is adapted from Keppenne and Rienecker (2002) where a massively parallel multivariate EnKF algorithm is derived and applied to the assimilation of in situ temperature data into a Pacific basin configuration of the same OGCM (Poseidon: Schopf and Loughe, 1995). With more than 30 million prognostic state variables, the global model's state-vector size restricts the ensemble size and limits the choice of systematic-error covariance modeling approaches. Accordingly, the bias covariances are derived from the same ensemble distribution as the random-error covariances, albeit different covariance scales are used in each covariance model. Also, a steady state model is assumed for the bias, but the bias estimate is allowed to propagate between successive assimilations (i.e. $\boldsymbol{b}_{k}^{f}=\boldsymbol{b}_{k-1}^{a}$ ).

The remainder of this article is organized as follows. Section 2 focuses on the methodology with a description of the model and assimilation system. In Sect. 3, the OBC algo- rithm is applied in the framework of T/P-anomaly assimilation with the Poseidon OGCM and the impact on seasonal hindcasts of using the EnKF to initialize the GMAO coupled general circulation model (CGCM) is examined. Temperature data are also assimilated but $\mathrm{OBC}$ is not used to process them. Section 4 contains the conclusion.

\section{Model and algorithms}

\subsection{Model}

The Poseidon model (Schopf and Loughe, 1995; Konchadi et al., 1998; Yang et al., 1999) is a finite-difference, reducedgravity ocean model which uses a generalized vertical coordinate designed to represent a turbulent, well-mixed surface layer and nearly isopycnal deeper layers. The prognostic variables are layer thickness, $h(\lambda \theta, \zeta, t)$, temperature, $T(\lambda \theta, \zeta, t)$, salinity, $S(\lambda \theta, \zeta, t)$, and the zonal and meridional current components, $u(\lambda \theta, \zeta, t)$ and $v(\lambda \theta, \zeta, t)$, where $\lambda$ is longitude, $\theta$ latitude, $t$ time and $\zeta$ is an isopycnal vertical coordinate. The SSH field is diagnostic.

Vertical mixing is parameterized through a Richardson number-dependent mixing scheme (Pacanowski and Philander, 1981) implemented implicitly. An explicit mixed layer is embedded within the surface layers following Sterl and Kattenberg (1994). For layers within the mixed layer, the vertical mixing and diffusion are enhanced to mix the layer properties through the depth of the diagnosed mixed layer. A time-splitting integration scheme is used whereby the hydrodynamics are done with a short time step $(15 \mathrm{~min})$, but the vertical diffusion, convective adjustment and filtering are done with coarser time resolution (half-daily).

The model's parallel implementation, described in Konchady et al. (1998), uses a message-passing protocol and a 2D horizontal domain decomposition.

The 27-layer configuration of the OGCM used here is the oceanic component of the coupled ocean-atmosphere-land model used in the GMAO seasonal-to-interannual forecasting system. Uniform $5 / 8^{\circ}$ zonal and $1 / 3^{\circ}$ meridional resolutions are used, resulting in $576 \times 538 \times 27$ grid boxes, of which $28 \%$ are situated over land. Thus, there are about $3.3 \times 10^{7}$ individual prognostic variables.

\subsection{Localized ensemble Kalman filter}

The EnKF has quickly gained popularity since its introduction by Evensen (1994). Applications range from simplemodel studies to uses with more realistic forecast models. Evensen (2003) lists most recent meteorological and oceanographic EnKF applications. For the Poseidon model, the parallel implementation is described in Keppenne and Rienecker (2002) and detailed in a NASA Technical Report (Keppenne and Rienecker, 2001). It is applied to the assimilation of in situ temperature observations into a Pacific basin version of Poseidon in Keppenne and Rienecker (2003). A less costly, steady-state version of the algorithm (multivariate optimal interpolation) is also discussed in Borovikov et al. (2005). 


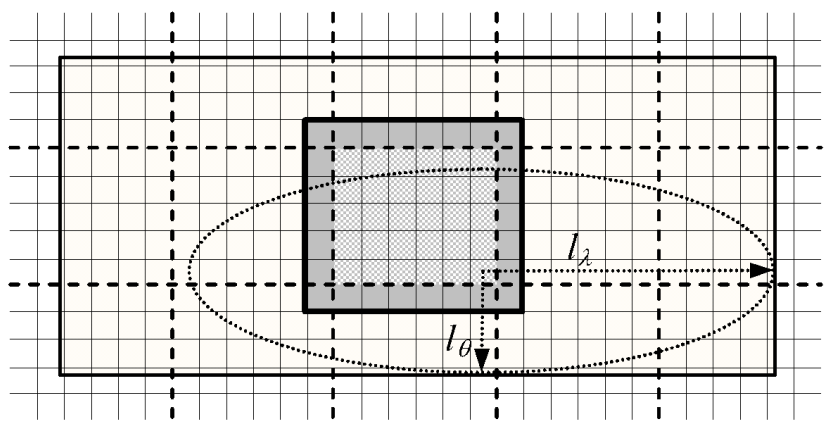

Fig. 1. Schematic view of the horizontal domain decomposition showing, for one PE, its PE-private area (textured inner rectangle), the halo region it shares with the surrounding PEs (darker shaded area), the ellipse with semi-axes $l_{\lambda}$ and $l_{\theta}$ which delimits the influence region of the PE-private area's southeastern corner cell (dotted) and the rectangular region from which it collects observations to assimilate (outer shaded rectangle containing the elliptical influence regions of all this PE's private grid cells). The dashed gridlines delimit the PE-private areas of nearby PEs. In the actual model, there are $576 \times 538 \times 27$ grid boxes, the PE-private areas have either $36 \times 33 \times 27$ or $36 \times 34 \times 27$ grid boxes and the halo regions are one cell wide.

To avoid costly ensemble transpositions across processors, the assimilation uses the same horizontal domain decomposition used to integrate the ensemble. Thus, the ensemble is distributed so that the memory of each processing element (PE) contains the same elements of each ensemble member's state vector. Although between 16 and 64 HP SC45 PEs are commonly used for single-model runs, a $16 \times 16 \mathrm{PE}$ lattice is used when running the EnKF. On this lattice, each PE is responsible for the assimilation of the observations that occur within a region surrounding its rectangular PE's private area. This region's size is a function of $l_{\lambda}$ and $l_{\Phi}$, two correlationfunction parameters specifying the distance at which a Schur product of the ensemble-derived covariances with a compactly supported correlation function forces background covariances to vanish in the zonal and meridional directions, respectively (Houtekamer and Mitchell, 2001; Keppenne and Rienecker, 2002). This is illustrated in Fig. 1.

For the sake of brevity, the parallelization of the EnKF is not explicitly discussed further in this paper and the equations are written as though a single processor were performing all the computations. For details, the reader is referred to Keppenne and Rienecker (2002).

Assume that the true climate state, $\boldsymbol{x}^{t}$, evolves from time $t_{k-1}$ to time $t_{k}$ according to ${ }^{2}$

$\boldsymbol{x}_{k}^{t}=\boldsymbol{M}\left(\boldsymbol{x}_{k-1}^{t}, \boldsymbol{f}_{k-1}\right)+\boldsymbol{\eta}_{k-1}$,

where $\boldsymbol{M}$ is the model operator, $\boldsymbol{f}_{k-1}$ is the forcing between $t_{k-1}$ and $t_{k}$, and $\boldsymbol{\eta}_{k-1}$, commonly referred to as system noise

\footnotetext{
${ }^{2}$ Upper-case boldface characters refer to matrices while lowercase boldface characters correspond to column vectors. Thus, given the vectors $\boldsymbol{a}$ and $\boldsymbol{b}, \boldsymbol{a}^{T} \boldsymbol{b}$ is their scalar product and $\boldsymbol{a} \boldsymbol{b}^{T}$ is their outer product.
}

or process noise, represents the effect of model imperfections and forcing errors between $t_{k-1}$ and $t_{k}$. The observations at time $t_{k}, \boldsymbol{y}_{k}$, are related to the true state by

$\boldsymbol{y}_{k}=\boldsymbol{H}_{k}\left(\boldsymbol{x}_{k}^{t}\right)+\boldsymbol{\varepsilon}_{k}$,

where $\boldsymbol{H}_{k}$ is the observation operator and $\varepsilon$ is a vector of observational errors. The model and observations are assumed unbiased so that $E\left(\boldsymbol{\eta}_{k-1}\right)=0$ and $E\left(\boldsymbol{\varepsilon}_{k}\right)=0$.

The Kalman filter update equation (Kalman, 1960) relates the analysis to the model forecast with

$\boldsymbol{x}_{k}^{a}=\boldsymbol{x}_{k}^{f}+\boldsymbol{K}_{k}\left[\boldsymbol{y}_{k}-\boldsymbol{H}_{k}\left(\boldsymbol{x}_{k}^{f}\right)\right]$,

where $\boldsymbol{K}_{k}$ is known as the Kalman gain and is given by

$\boldsymbol{K}_{k}=\boldsymbol{P}_{k}^{f} \boldsymbol{H}_{k}^{T}\left[\boldsymbol{H}_{k} \boldsymbol{P}_{k}^{f} \boldsymbol{H}_{k}^{T}+\boldsymbol{R}_{k}\right]^{-1}$.

The matrices $\boldsymbol{P}_{k}^{f}$ and $\boldsymbol{R}_{k}$ are the background-error and observational-error covariances, respectively. With the notable exception of particle methods (e.g. Anderson and Anderson, 1999; Van Leeuwen, 2003) in which the calculation of the Kalman gain is not necessary, all sequential data assimilation methods use an analysis equation like Eq. (3), but they differ in the assumptions that are made regarding $\boldsymbol{P}_{k}^{f}$ and $\boldsymbol{R}_{k}$. In the EnKF, an ensemble of $n$ model state vectors is evolved from $t_{k-1}$ to $t_{k}$ owing to

$\boldsymbol{x}_{i, k}^{f}=\boldsymbol{M}\left(\boldsymbol{x}_{i, k-1}^{a}, \boldsymbol{f}_{k-1}\right)+\boldsymbol{N}_{i, k-1}, \quad i=1,2, \ldots, n$,

where the first subscript refers to the $i$ th ensemble member and $N_{i, k-1}$ is a term added to the model equations to simulate the process noise. Many applications ignore the processnoise model and attempt to represent its effect through covariance inflation, but our experience with the Poseidon model suggests that traditional multiplicative covariance inflation may not be a viable approach when working with a high-dimensional state vector.

Since the remainder of the discussion refers to time $t_{k}$, the corresponding subscript will now be dropped except where required. Given the ensemble, $\left\{\boldsymbol{x}_{1}^{f}, \boldsymbol{x}_{2}^{f}, \ldots, \boldsymbol{x}_{n}^{f}\right\}$, of model forecasts, the EnKF first estimates $\boldsymbol{H} \boldsymbol{P}^{f} \boldsymbol{H}^{T}$ with

$$
\begin{aligned}
& \boldsymbol{S}=\left\{\boldsymbol{s}_{1}, \boldsymbol{s}_{2}, \ldots, \boldsymbol{s}_{n}\right\}=\left\{\boldsymbol{H}\left(\boldsymbol{x}_{1}^{f}\right), \boldsymbol{H}\left(\boldsymbol{x}_{2}^{f}\right), \ldots, \boldsymbol{H}\left(\boldsymbol{x}_{n}^{f}\right)\right\} \\
& \boldsymbol{H} \boldsymbol{P}^{f} \boldsymbol{H}^{T}=\frac{1}{n-1} \boldsymbol{S} \boldsymbol{S}^{T} .
\end{aligned}
$$

Then, the representer weights used to update the $i$ th ensemble member are calculated with

$\boldsymbol{a}_{i}=\left[\boldsymbol{C} \bullet \boldsymbol{H} \boldsymbol{P}^{f} \boldsymbol{H}^{T}+\boldsymbol{R}\right]^{-1}\left(\boldsymbol{y}+\boldsymbol{e}_{i}-\boldsymbol{H}\left(\boldsymbol{x}_{i}^{f}\right)\right)$,

where $\boldsymbol{C}$ is a compactly supported correlation matrix as discussed in Keppenne and Rienecker (2002), $\boldsymbol{e}_{i}$ is a perturbation term with covariance $\boldsymbol{R}$ (Burgers et al., 1998) and $\bullet$ represents the Schur (element by element) product (Houtekamer 
and Mitchell, 2001). Then, in each grid box $l$, with coordinates $\left(\lambda_{l}, \theta_{l}, \zeta_{l}\right)$, the state variables of ensemble member $i$ are updated using

$\boldsymbol{x}_{i, l}^{a}=\boldsymbol{x}_{i, l}^{f}+\frac{1}{n-1} \sum_{j=1}^{n} \boldsymbol{x}_{j, l}^{f} \boldsymbol{s}_{j}^{T}\left(\boldsymbol{c}_{l} \bullet \boldsymbol{a}_{i}\right)$,

where the rightmost term is computed right to left to reduce the computational burden, $\boldsymbol{x}_{i, l}=\left\{h_{i, l}, T_{i, l}, S_{i, l}, u_{i, l}, v_{i, l}\right\}$ and $c_{l}$ is a vector of correlation function coefficients. Denoting the difference between the longitudes, latitudes and vertical coordinates of grid box $l$ and the $m$ th observation as $\boldsymbol{\delta}_{l m}=\left(\delta \lambda_{l m}, \delta \theta_{l m}, \delta \zeta_{l m}\right)$, the $m$ th component of $\boldsymbol{c}_{l}$ is given by

$c_{l, m}=\rho\left(\boldsymbol{\delta}_{l m}\right)=\rho_{\lambda}\left(\delta \lambda_{l m}\right) \rho_{\theta}\left(\delta \theta_{l m}\right) \rho_{\zeta}\left(\delta \zeta_{l m}\right)$.

In Eq. (9), $\rho_{\lambda}, \rho_{\theta}$ and $\rho_{\zeta}$ represent applications of a compactly supported correlation function (Eq. 4.10 of Gaspari and Cohn, 1995) with scaling such that the correlations vanish at $d_{\lambda}, d_{\theta}$ and $d_{\zeta}$ in the respective space dimensions. The elements of $\boldsymbol{C}$ in Eq. (7) are obtained similarly, but are functions of coordinate differences between pairs of observations.

In the current study, the system-noise is modeled by adding to the model equations for each ensemble member a perturbation term computed as a linear combination of scaled empirical orthogonal functions (EOFs) calculated offline from an ensemble of ocean states forced with an ensemble of atmospheric forcing sets with perturbations representing internal atmospheric noise (Borovikov et al., 2005). The amplitude of the perturbations is continually adjusted during the ensemble integration in an effort to ensure

$\operatorname{tr}\left(\boldsymbol{H} \boldsymbol{P}^{f} \boldsymbol{H}^{T}+\boldsymbol{R}\right) \cong\left(\boldsymbol{y}-\boldsymbol{H} \overline{\boldsymbol{x}}^{f}\right)^{T}\left(\boldsymbol{y}-\boldsymbol{H} \overline{\boldsymbol{x}}^{f}\right)$.

The approximate equality is maintained as follows. If at time $t_{k}$ the left hand side of Eq. (10) is smaller (vs. greater) than the right hand side term by $25 \%$ or more, the amplitude of the perturbations applied between $t_{k}$ and $t_{k+1}$ is increased (vs. decreased) by $25 \%$. Note that this trick is analogous in its purpose to traditional covariance inflation.

Although the observation perturbation term, $\boldsymbol{e}_{i}$ in Eq. (7), can be avoided if a square-root formulation of the EnKF (e.g. Tippett et al., 2003) is used, a square-root algorithm is very inefficient in a localized analysis framework with compactly supported covariances. The reason for this is that the squareroot alternative to Eq. (8) would involve the solution of an $n \times n$ eigenproblem in each grid box. Nevertheless, provided that $\sum_{i=1}^{n} \boldsymbol{e}_{i}=\mathbf{0}$, the ensemble-mean update is unaffected by the perturbed observations.

\subsection{Bias estimation}

The previous discussion assumes that the forecast errors and observational errors are unbiased. While the latter assumption is plausible given that the measurements can be biascorrected when the instruments are known to produce systematic errors, the former is more questionable. The approach used here to account for the presence of forecastmodel bias is adapted from Dee and Todling (2000).
In analogy to Eq. (1), the true model bias is assumed to evolve according to

$\boldsymbol{b}_{k}^{t}=\boldsymbol{M}_{b}\left(\boldsymbol{b}_{k-1}^{t}, \boldsymbol{x}_{k-1}^{t}\right)+\boldsymbol{\Omega}_{k-1}$,

where $\boldsymbol{\Omega}_{k-1}$ is a white noise process. Since the bias evolution is much slower than that of the forecast and happens on much longer timescales, a reasonable model for the $\boldsymbol{M}_{b}$ operator is the identity matrix. Similarly, $\boldsymbol{\Omega}_{k-1}$ is neglected because we assume that $\boldsymbol{\Omega}_{k-1}<<\boldsymbol{\eta}_{k-1}$. Therefore, the augmented statepropagation equations are

$\boldsymbol{x}_{i, k}^{f}=\boldsymbol{M}\left(\boldsymbol{x}_{i, k-1}^{a}, \boldsymbol{f}_{k-1}\right)+\boldsymbol{N}_{i, k-1}, \quad i=1, \ldots, n$,

$\boldsymbol{b}_{k}^{f}=\boldsymbol{b}_{k-1}^{a}$

The unbiased state estimate is $\overline{\boldsymbol{x}}^{f}-\boldsymbol{b}^{f}$, where $\overline{\boldsymbol{x}}$ refers to the ensemble mean. Therefore and in analogy to Eq. (3), the two-stage update proceeds as follows:

$\boldsymbol{b}_{k}^{a}=\boldsymbol{b}_{k}^{f}-\boldsymbol{L}_{k}\left[\boldsymbol{y}_{k}-\boldsymbol{H}_{k}\left(\overline{\boldsymbol{x}}_{k}^{f}-\boldsymbol{b}_{k}^{f}\right)\right]$,

$\boldsymbol{x}_{i, k}^{a}=\boldsymbol{x}_{i, k}^{f}+\boldsymbol{K}_{k}\left[\boldsymbol{y}_{k}+\boldsymbol{e}_{i}-\boldsymbol{H}_{k}\left(\boldsymbol{x}_{i, k}^{f}-\boldsymbol{b}_{a}^{f}\right)\right], \quad i=1, \ldots, n$.

The state update (Eq. 13b) is computed and applied as in Eqs. (6-8), except that the unbiased state estimate, $\boldsymbol{x}_{i}^{f}-\boldsymbol{b}^{a}$, is used everywhere in lieu of $\boldsymbol{x}_{i}^{f}$. The gain for the bias, given by $\boldsymbol{L}=\boldsymbol{P}_{b}^{f} \boldsymbol{H}^{T}\left[\boldsymbol{H} \boldsymbol{P}_{b}^{f} \boldsymbol{H}^{T}+\boldsymbol{R}\right]^{-1}$, is preceded by a minus sign in Eq. (13a) because a systematically positive assimilation increment suggests the presence of a negative model bias. Note that it has been assumed that the bias and the unbiased errors are uncorrelated. The bias update uses equations similar to Eqs. (6-8):

$$
\begin{aligned}
& \boldsymbol{S}_{b}=\left\{\boldsymbol{H}\left(\boldsymbol{x}_{1}^{f}-\boldsymbol{b}^{f}\right), \boldsymbol{H}\left(\boldsymbol{x}_{2}^{f}-\boldsymbol{b}^{f}\right), \ldots, \boldsymbol{H}\left(\boldsymbol{x}_{n}^{f}-\boldsymbol{b}^{f}\right)\right\}, \\
& \boldsymbol{H} \boldsymbol{P}_{b}^{f} \boldsymbol{H}^{T}=\frac{\alpha}{n-1} \boldsymbol{S}_{b} \boldsymbol{S}_{b}^{T}, \\
& \boldsymbol{a}_{b}=\left[\boldsymbol{C}_{b} \bullet \boldsymbol{H} \boldsymbol{P}_{b}^{f} \boldsymbol{H}^{t}+\boldsymbol{R}\right]^{-1}\left(\boldsymbol{y}-\boldsymbol{H}\left(\overline{\boldsymbol{x}}^{f}-\boldsymbol{b}_{f}\right)\right), \\
& \boldsymbol{b}_{l}^{a}=\boldsymbol{b}_{l}^{f}-\frac{\alpha}{n-1} \sum_{j=1}^{n} \boldsymbol{b}_{j, l}^{f} \boldsymbol{s}_{j}^{T}\left(\boldsymbol{c}_{b, l} \bullet \boldsymbol{a}_{b}\right),
\end{aligned}
$$

The $l$ subscript in Eq. (14d) refers to the $l$ th grid box. The $\alpha$ in Eq. (14b) and Eq. (14d) is an amplitude scaling factor. The correlation matrix $\boldsymbol{C}_{b}$ and the correlation vectors $\boldsymbol{c}_{b, l}$ are computed as $\boldsymbol{C}$ and $\boldsymbol{c}_{l}$, but using different correlation scales because the spatial scales are expected to be longer for the bias than for the random errors if the systematic model errors reflect large-scale atmospheric forcing biases. The adjustable parameters of the bias estimation algorithm are thus $\alpha$ and the spatial scales for the bias: $l_{\lambda, b}, l_{\theta, b}$ and $l_{\varsigma, b}$. 


\section{Application}

\subsection{Assimilation experiments}

Altimeter measurements from space are important because they provide insight into subsurface ocean dynamics such as changes in the vertical displacement of the thermocline and halocline layers. The T/P altimeter samples the SSH field with an error standard deviation of approximately $2 \mathrm{~cm}$. After removal of the temporal mean from repeating measurement tracks and of the specific frequency distribution of known tides, the residual time-varying measurements have a spatial resolution of about $1 \mathrm{~km}$ along the orbit track and $317 \mathrm{~km}$ between tracks at the equator. The orbit repeats itself every 9.9156 days.

The T/P data used in this study are processed as in Adamec (1998), resulting in an along-track average every $7 \mathrm{~km}$. Since temperature profiles are the most abundant source of information about subsurface variability, TAO and XBT profiles and, when available, temperature profiles from ARGO and PIRATA are also assimilated.

The assimilation of the altimeter observations into the OGCM presents a peculiar problem. Since the data are anomalies, the model climatology is used to reconstruct the signal prior to each assimilation. However, our previous experience with temperature and altimeter assimilation has indicated that the time-mean SSH fields from free-model and assimilation runs are markedly different. Moreover, when altimeter data are processed, the model gradually shifts regimes and the adjustment - which may require several months of simulation - has repercussions on the OMF calculations. When a static model climatology is added to the anomalies before they are fed to the assimilation system, the resulting signal tends to steer the model towards an unnatural state. The problem can be mitigated if bias correction is used in the altimetry assimilation and the SSH climatology is continuously adjusted as the measurements are processed.

The experimental setting as as follows. The model complexity and the specifications of the parallel computing platform used (HP SC45) restrict the ensemble size to 16-members. To minimize the effect of using small ensembles, the deviations, $\left(\boldsymbol{x}_{i}^{f}-\overline{\boldsymbol{x}}^{f}\right), i=1, \cdots, n$, are heavily pre-filtered by means of a spatial averaging in which the weights are proportional to $\exp \left(-\right.$ distance $\left.^{2}\right)$, before the required background covariances are calculated. The prefiltering After a long experimentation, the spatial scales used in the Schur-product calculations are $d_{\lambda}=60^{\circ}, d_{\theta}=30^{\circ}$ and $d_{\varsigma}=400 \mathrm{~m}$ in the temperature assimilation. When altimeter data are processed, $d_{\lambda}=20^{\circ}, d_{\theta}=10^{\circ}$ and $d_{\zeta}=400 \mathrm{~m}$. The assimilation interval is five days, but the SSH and temperature data are processed in sequence. The separate processing of the two data types is justified by the lack of correlation between the temperature and altimeter observational errors. Incremental analysis updating (IAU: Bloom et al., 1996), in which the analysis increments are applied gradually (divided equally between time steps) over the sequence of time steps from $t_{k}$ to $t_{k+1}$, is used in all runs.
The observational-error covariance matrices, $\boldsymbol{R}$, are assumed to be Gaussian and are modeled with the same compactly supported correlation function used in the Schur product calculations. For the T/P data, an observational errorvariance of $4 \mathrm{~cm}^{2}$ is assumed and the data-error covariances are forced to vanish at a distance of $10^{\circ}$. For the temperature observations, the assumed error-variance is $0.25 \mathrm{C}^{2}$, and covariances vanish at $20^{\circ}$ horizontally and - reflecting the belief that the errors are strongly correlated within each temperature profile - at a spacing of $1000 \mathrm{~m}$ vertically.

In all experiments, the initial conditions come from a freemodel run forced with observed winds, heat fluxes and SSTs, but with no data assimilation. The initial ensemble is constructed around the state from the free-model run which we identify as ensemble member 1 . The ensemble construction is based on perturbations of the form

$\boldsymbol{x}_{i}=\boldsymbol{x}_{1}+\sum_{j} \gamma_{i j} \boldsymbol{v}_{j}, \quad i=2, \cdots, n$,

where the $\gamma_{i j}$ are normally distributed pseudorandom numbers and the $\boldsymbol{v}_{j}$ are the leading EOFs of an ensemble of ocean runs calculated as in Borovikov et al. (2005). Given that the higher-order EOFs reflect variability on smaller spatial scales, thirty two EOFs are used as a compromise between using just as many EOFs as there are ensemble members (to guarantee a minimum rank) and using all available EOFs (to cover all the spatial scales of the model variability). The perturbations are applied gradually over a period of ten days using the same IAU procedure used to insert the assimilation increments. Thereafter, the same equation and insertion procedure are used daily to generate and apply perturbations to simulate the system noise - always striving to respect the condition (Eq. 10).

In both the temperature and altimetry assimilations, crossfield covariances are used to update $T, S, u$ and $v$ in each grid box. The layer thicknesses are not updated and adjust freely between successive assimilations in response to the IAU. Bias correction is not used when temperature is assimilated. When OBC is used in the altimetry assimilation, adjustments are continuously made to the SSH climatology but the $T, S, u, v$ and $h$ fields are not bias-corrected.

Although insight into the value of the parameters, $\alpha, d_{\lambda, b}$, $d_{\theta, b}$ and $d_{\varsigma, b}$, of the OBC algorithm can be gained from an analysis of OMF statistics, these parameters are adjusted in a series of short tuning runs. First, the bias-covariance scales were set to the same value as the corresponding scales for the background covariances - i.e. $d_{\lambda, b}, d_{\theta, b}=d_{\theta}$, and $d_{\varsigma, b}=d_{\varsigma}-$ and $\alpha$ is varied in the interval $(0-2)$. For each probed value of $\alpha$, the T/P assimilation ran for two months starting on 1 January 1993 and the RMS OMF for SSH was calculated for the entire run. The results, shown in Table 1, prompted us to choose $\alpha=0.75$ for the remaining experiments.

To simplify the task of tuning the bias-covariance scales, a parameter, $\beta$, is introduced such that $d_{\lambda, b}=\beta d_{\lambda}, d_{\theta, b}=\beta d_{\theta}$ and $d_{\varsigma, b}=\beta d_{\varsigma}$. The RMS OMF statistics of a new series of two-month runs varying $\beta$ with $\alpha$ fixed at 0.75 lead to the choice, $\beta=3.0$ (Table 2 ). Of course, the procedure followed to 
Table 1. RMS OMF of the unbiased estimate for SSH as a function of $\alpha$ in the runs with $d_{\lambda, b}, d_{\theta, b}=d_{\theta}$, and $d_{\zeta, b}=d_{\varsigma}$.

\begin{tabular}{lllllllll}
\hline$\alpha$ & 0.0 & 0.1 & 0.25 & 0.5 & 0.75 & 1.0 & 1.5 & 2.0 \\
RMS (m) & 0.087 & 0.084 & 0.081 & 0.074 & 0.072 & 0.089 & 0.104 & 0.127 \\
\hline
\end{tabular}

Table 2. RMS OMF of the unbiased estimate for SSH as a function of $\beta$ in the runs with $\alpha=0.75$.

\begin{tabular}{lllllll}
\hline$\beta$ & 0.5 & 1.0 & 2.0 & 3.0 & 4.0 & 5.0 \\
RMS (m) & 0.087 & 0.072 & 0.066 & 0.061 & 0.063 & 0.065 \\
\hline
\end{tabular}
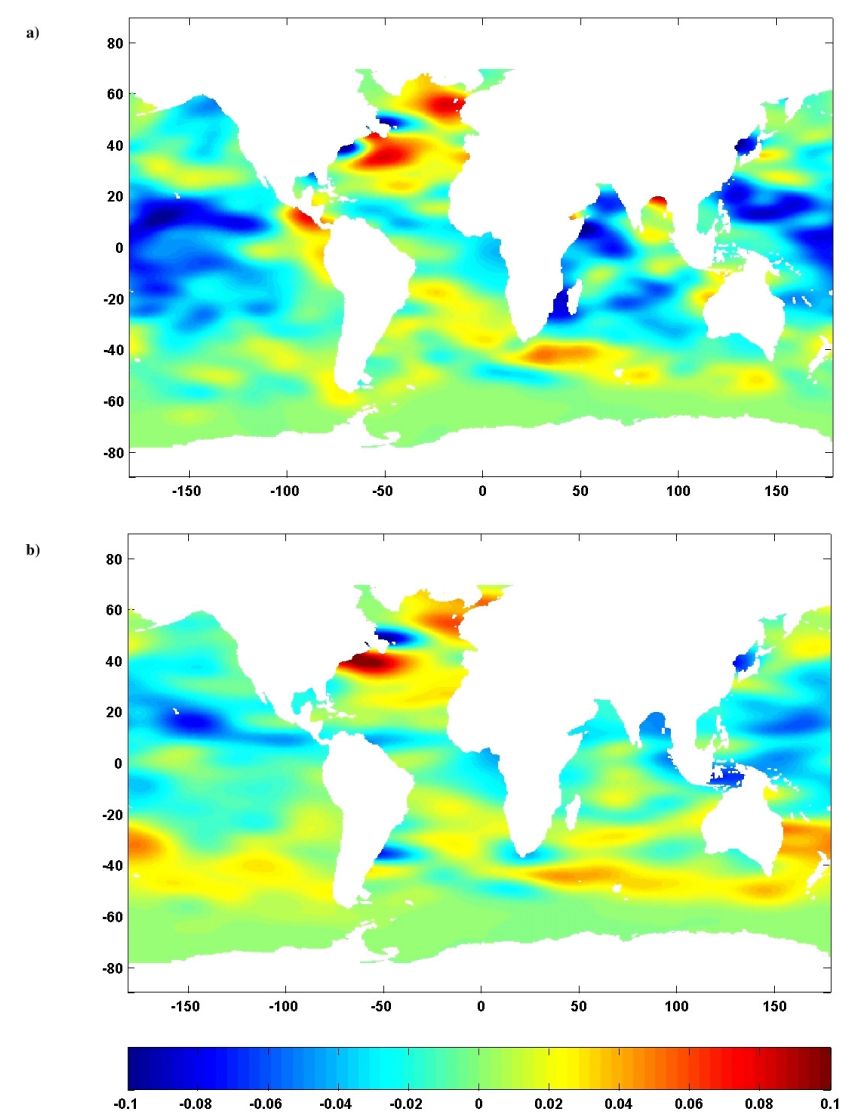

Fig. 2. Estimates of the SSH climatology error on (a) 1 July 1993 and (b) 1 January 1994, respectively after six and twelve months of temperature $+\mathrm{T} / \mathrm{P}$ altimetry assimilation with bias correction in the EnKF-OBC run.

adjust $\alpha$ and $\beta$ - by first searching for a value of $\alpha$ that minimizes RMS OMF statistics when $\beta=1$ before varying $\beta$ while keeping $\alpha$ at this value - in no way assures that the resulting $(\alpha, \beta)$ pair will minimize RMS statistics. Further iterations with $\beta=3.0$ and varying $\alpha$ would likely yield a "better" $(\alpha$, $\beta)$ pair.
The next experiment is a comparison of three 12-month runs, starting 1 January 1993. In the first run (EnKF-OBC), both temperature and $\mathrm{T} / \mathrm{P}$ data are assimilated, and $\mathrm{OBC}$ is used in the T/P assimilation to continually adjust the $\mathrm{SSH}$ climatology (experience has shown that the misspecification of the SSH climatology is a more serious problem than the presence of a bias in the temperature, salinity or current fields and no attempt is made to correct the temperature, salinity or current bias in this run.). The second run (EnKF) is identical to the first, except that OBC is not used, and the third run (control) is a free-model run without assimilation.

Figure 2 shows the $\mathrm{SSH}$ bias estimate at six and twelve months in the EnKF-OBC run. The differences between the two estimates can likely be attributed to the continuing convergence of the $\mathrm{OBC}$ algorithm as well as to the presence of seasonality in the bias. A persistent feature of the bias estimate discernable in Figs. $2 \mathrm{a}$ and $2 \mathrm{~b}$ is a positive bias in areas of the North Atlantic and in the southern midlatitudes. Western boundary current overshoots in the subtropics and subpolar regions are a likely cause for the positive bias in the North Atlantic. The mostly negative bias in the Tropical Pacific occurs between the latitudes of zero wind stress curl and is probably associated with the SSM/I forcing.

\subsection{Background covariances}

One hallmark feature of the EnKF is its reliance on flow dependent background covariances in the Kalman gain calculation. In many operational assimilation systems, the contribution of a single additional observation to the assimilation increment is solely a function of the corresponding innovation, $\boldsymbol{y}-\boldsymbol{H}\left(\boldsymbol{x}^{f}\right)$. The background covariances are often univariate and isotropic, do not vary with time and - if at all dependent on the measurement location - the spatial dependency is usually very simple and specified a priori (in analogy with microeconomics, we refer to the contribution of a single observation to the Kalman gain as that observation's marginal gain.).

With the EnKF, the marginal gain of a unit innovation results from the local (in space and time) ensemble configuration in the neighborhood of the corresponding measurement. 

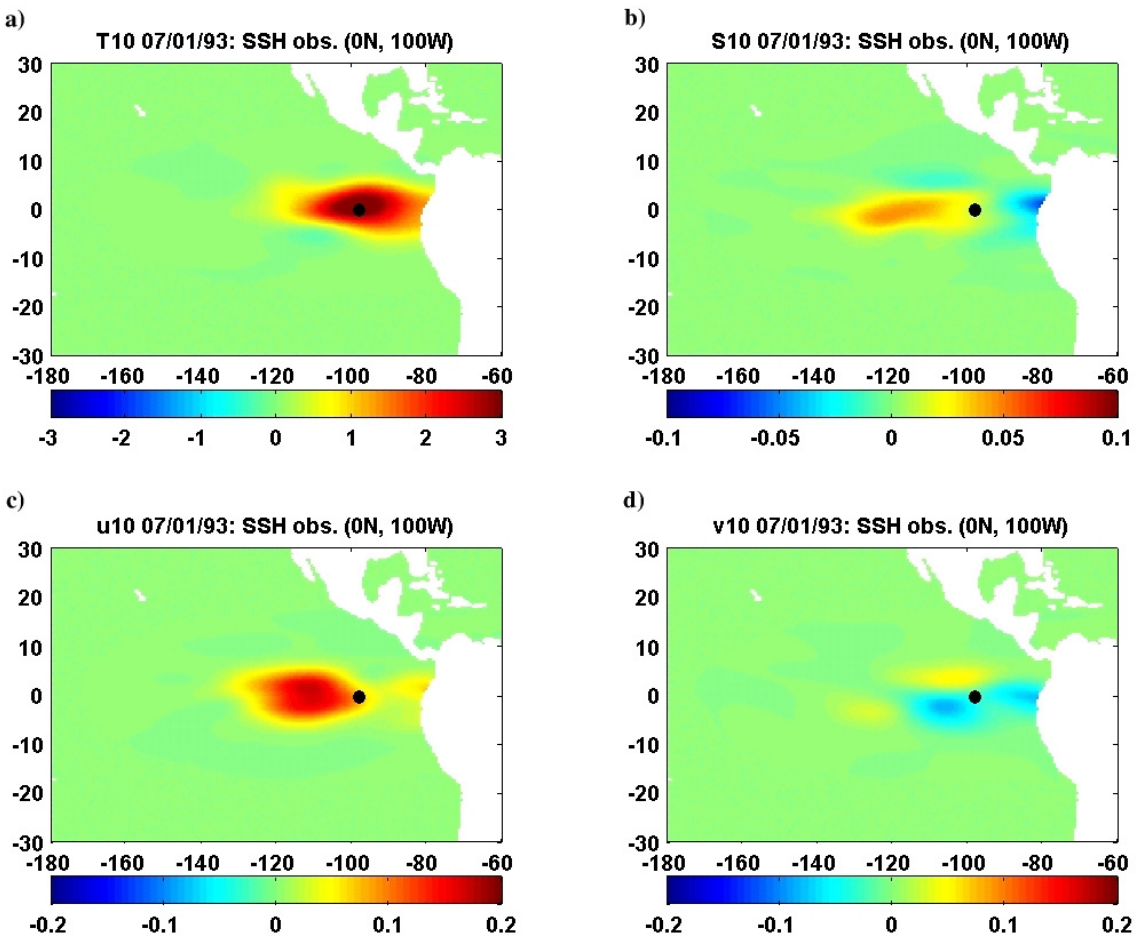

Fig. 3. Isopycnal cross-sections showing layer 10 of the Marginal Kalman gain corresponding to a hypothetical $0.1 \mathrm{~m}$ SSH innovation at $\left(0^{\circ} \mathrm{N}, 100^{\circ} \mathrm{W}\right)$ on 1 July 1993 . The large dot corresponds to the latitude and longitude of the observation. (a) $T$ gain, (b) $S$ gain, (c) $u$ gain and (d) $v$ gain.

Figures 3-7, which all correspond to the EnKF-OBC run, illustrate this point.

In Fig. 3, we show the marginal contribution on 1 July 1993 of a hypothetical $0.1 \mathrm{~m} \mathrm{SSH}$ innovation at $\left(0^{\circ} \mathrm{N}\right.$, $100^{\circ} \mathrm{W}$ ) to the $T, s, u$ and $v$ increments in layer 10 (counting from the surface). The anisotropy of the background covariances is evident. The positive SSH innovation results in a warmer layer 10 and the temperature increase is centered around the SSH observation (Fig. 3a). In contrast, $S$ corrections increase west of the datum and decrease to the east (Fig. 3b). The zonal current of the South Equatorial Current decelerates, mostly west of the observation (Fig. 3c), and there is increased meridional divergence of the meridional current (Fig. 3d).

Figure 4 illustrates the flow dependency of the background covariances by showing how the depth of the $20^{\circ} \mathrm{C}$ isotherm is affected by the same $0.1 \mathrm{~m} \mathrm{SSH}$ innovations on $1 \mathrm{July}$ 1994 and on 1 January 1994. The positive SSH innovations result in a deeper thermocline in both cases, although on 1 July 1993 the deepening occurs mostly to the east of the observation location, while on 1 January 1994 it happens in a zonally elongated pattern centered about the datum.

The effect of the $0.1 \mathrm{~m} \mathrm{SSH}$ innovations at $\left(0^{\circ} \mathrm{N}, 100^{\circ} \mathrm{W}\right)$ on 1 July 1993 and 1 January 1994 on the SSH bias estimate is shown in Fig. 5. In general, a positive SSH innovation results in a deepening of the thermocline and in a negative SSH bias increment since the model is likely to be negatively biased when the observed SSH is consistently higher than what it predicts.
Because the bias update (Eq. 14) uses wider covariance localization scales than the state update, the marginal SSH bias increments cover a much larger geographical area. Moreover, while we never allow a datum to affect the ocean state in another basin (Pacific, Atlantic or Indian Ocean) than that where it is observed, we do not impose the same restriction on the bias increment. While some bias patterns result from errors in ocean model parameterization (e.g. Vossepoel et al., 2005), other patterns are believed to reflect large-scale atmospheric forcing biases. It is sensible to assume that the latter patterns will not be confined to a particular ocean. Accordingly, the zonally elongated negative bias increment in the eastern Equatorial Pacific extends in the Atlantic on 1 July 1993 (Fig. 5a). On 1 January 1994 (Fig. 5b), the bias increment is still mostly negative in the eastern Equatorial Pacific, but in the Tropical Atlantic it is generally opposite to the bias increment obtained on 1 July 1993.

Figure 6 shows isopycnal cross sections through layer 10 through the marginal $T, s, u$ and $v$ increments corresponding to a positive $1^{\circ} \mathrm{C}$ innovation in the Western Pacific at $\left(20^{\circ} \mathrm{N}, 150^{\circ} \mathrm{E}\right)$ at a depth of $53 \mathrm{~m}$ (layer 10$)$. The positive temperature innovation produces a salinity increase to its north and a freshening to its south (Figs. 6a and 6b). As seen in Fig. 3 for the SSH innovations in the eastern Equatorial Pacific, the marginal $u$ and $v$ gains have generally opposite signs (Figs. 6c and 6d).

The background covariances estimated with the EnKF exhibit not only anisotropy and temporal variations, but also a strong dependency on the observation location. To illustrate 

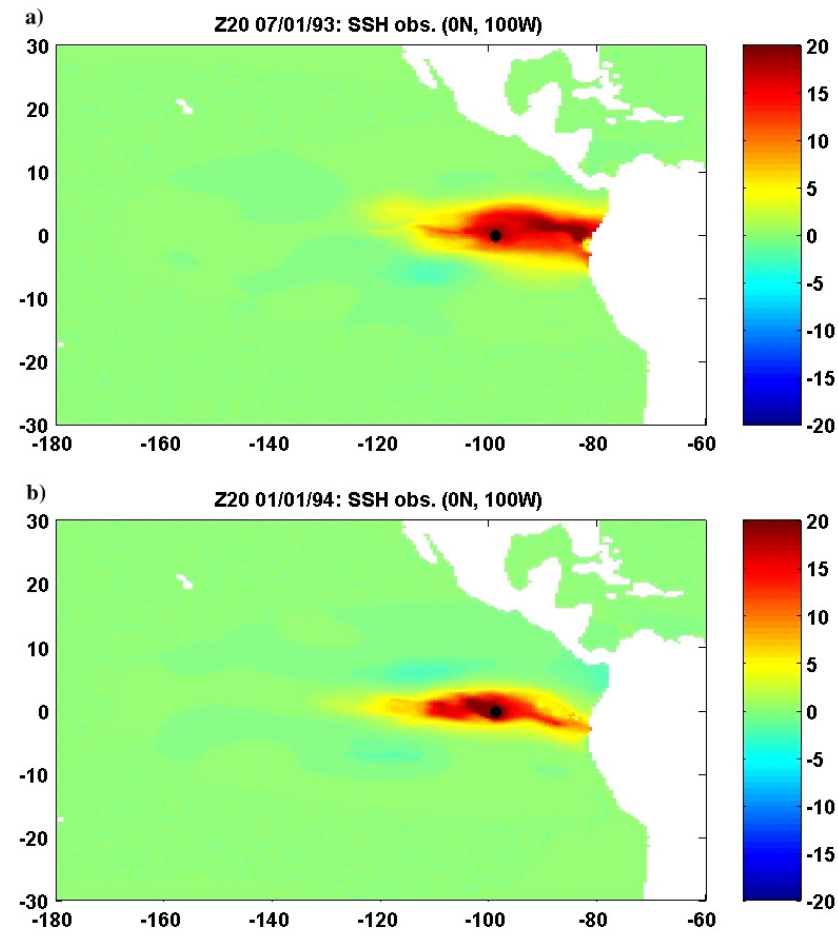

Fig. 4. Change in the depth of the $20^{\circ} \mathrm{C}$ isotherm in response to a hypothetical $0.1 \mathrm{~m} \mathrm{SSH}$ innovation on (a) 1 July 1993 and (b) 1 January 1994.

this, Fig. 7 shows layer 10 of the $T, s, u$ and $v$ increments corresponding to a $1{ }^{\circ} \mathrm{C}$ innovation at $\left(20^{\circ} \mathrm{S}, 60^{\circ} \mathrm{E}\right)$ and at a depth of $68 \mathrm{~m}$ (layer 10 at $20^{\circ} \mathrm{S}, 60^{\circ} \mathrm{E}$ ) on 1 July 1993. Here, the temperature increase in layer 10 caused by the $1^{\circ} \mathrm{C}$ innovation (Fig. 7a) is larger than in the case of the Western Pacific innovation (Fig. 6a). Moreover, the temperature increase extends more to the south of the observation than to its north. The amplitude of the marginal $u$ gain is also much larger than in the case of the Western Pacific innovation and is positive east of the observation and negative north of it (Fig. 7c). On the other hand, the amplitude of the marginal $v$ gain is relatively small (Fig. 7d). In contrast to the case of the Western Pacific temperature observation (Fig. 6b), the marginal $S$ gain exhibits a wavy zonal pattern with freshening east and west of the datum and a salinity increase closer to it (Fig. 7b).

One important question which has not been addressed in this study because of computational limitations is whether marginal Kalman gains like those shown in Figs. 6 and 7 are robust with respect to increases in ensemble size. Before this is done, we can not rule out the presence of artificial biases in the corrections. Also note that the EnKF correction is variance minimizing only for Gaussian ensemble distributions (van Leeuwen, 2001).

\subsection{OMF statistics}

The SSH bias is a two-dimensional field. Notwithstanding and with the caveat that, in a spatial average, a large positive a) SSH bias incr. 07/01/93: SSH obs. (0N, 100W)
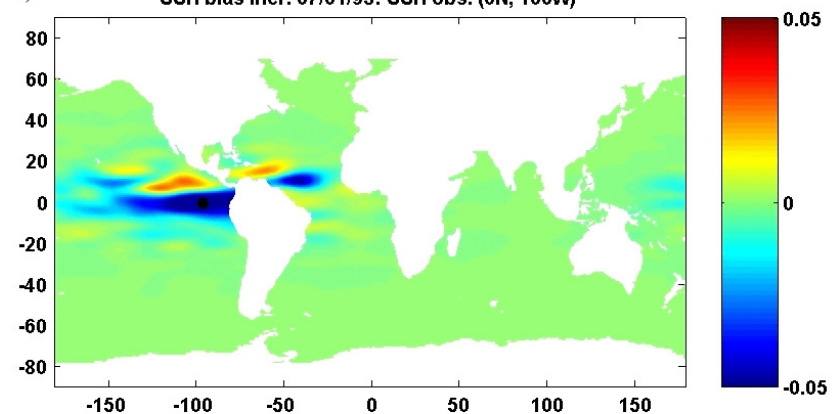

b)

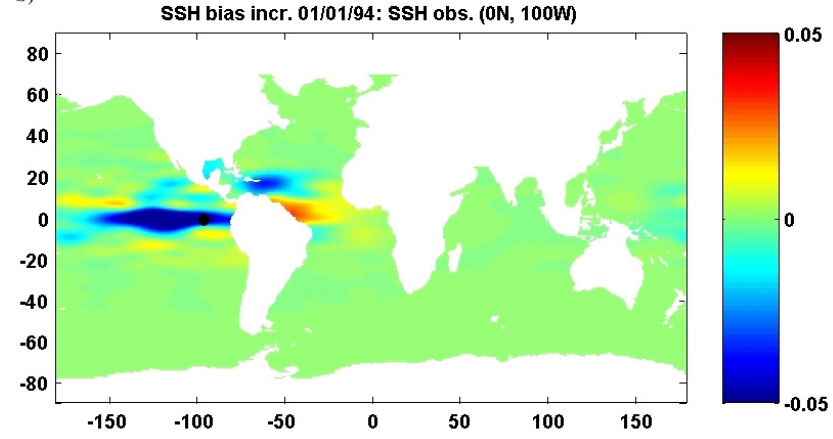

Fig. 5. Marginal Kalman gain for the SSH bias field corresponding to a hypothetical $0.1 \mathrm{~m} \mathrm{SSH}$ innovation on (a) 1 July 1993 and (b) 1 January 1994.

bias in one part of the ocean could cancel out a large negative bias in another part, insight into whether the assimilation reduces or eliminates systematic errors can be gained by examining time series of spatial-mean OMF statistics. Accordingly, Fig. 8 shows how the latter evolve during the 12-month experiment. The statistics shown lump all observations together, independent of depth. Since SSH is a diagnostic variable, improvements in its statistics mostly reflect improvements in the subsurface temperature and salinity fields.

In Fig. 8a, showing the OMF corresponding to the temperature observations, the strong positive bias of the control run appears to be quickly reduced, but not eliminated, in the EnKF-OBC and EnKF runs. In fact, although the original Kalman filter equations are not designed to handle bias, a significant part of the work done by the assimilation consists in correcting the bias. The same observation can be made about most ocean data assimilation systems. Since the temperature bias is not explicitly estimated or corrected in the EnKF and EnKF-OBC runs, the corresponding spatial-mean OMF differences do not show much difference. Averaged over the entire run, the spatial-mean OMF differences are $0.08^{\circ} \mathrm{C}, 0.10^{\circ} \mathrm{C}$ and $0.22^{\circ} \mathrm{C}$ respectively in the EnKF-OBC, EnKF and control runs. The situation is different for the spatial-mean SSH OMF (Fig. 8b). After an initial adjustment, the estimated unbiased OMF from the EnKF-OBC run is much closer to zero than the biased OMF from the same run, or than the spatial-mean OMF of the control and EnKF runs. Averaged over the entire run, the spatial-mean OMF is $0.0 \mathrm{~cm}, 0.6 \mathrm{~cm}, 0.6 \mathrm{~cm}$ and $0.12 \mathrm{~cm}$ for the unbiased EnKF- 

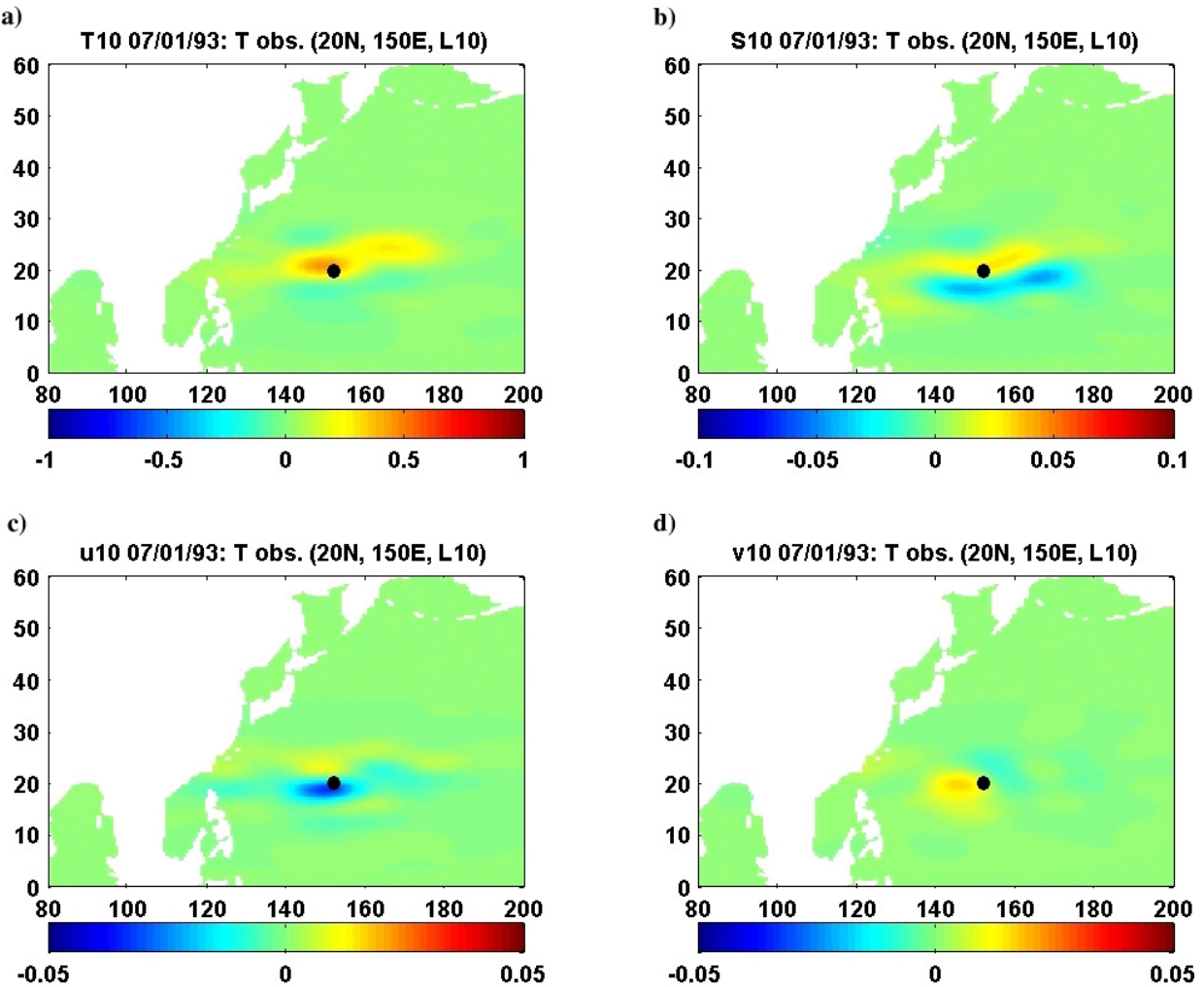

Fig. 6. Isopycnal cross sections showing layer 10 of the Marginal Kalman gain corresponding to a hypothetical $1^{\circ} \mathrm{C}$ temperature innovation in layer 10 at $\left(20^{\circ} \mathrm{N}, 150^{\circ} \mathrm{E}\right)$ on 1 July 1993. (a) $T$ gain, (b) $S$ gain, (c) $u$ gain and (d) $v$ gain.
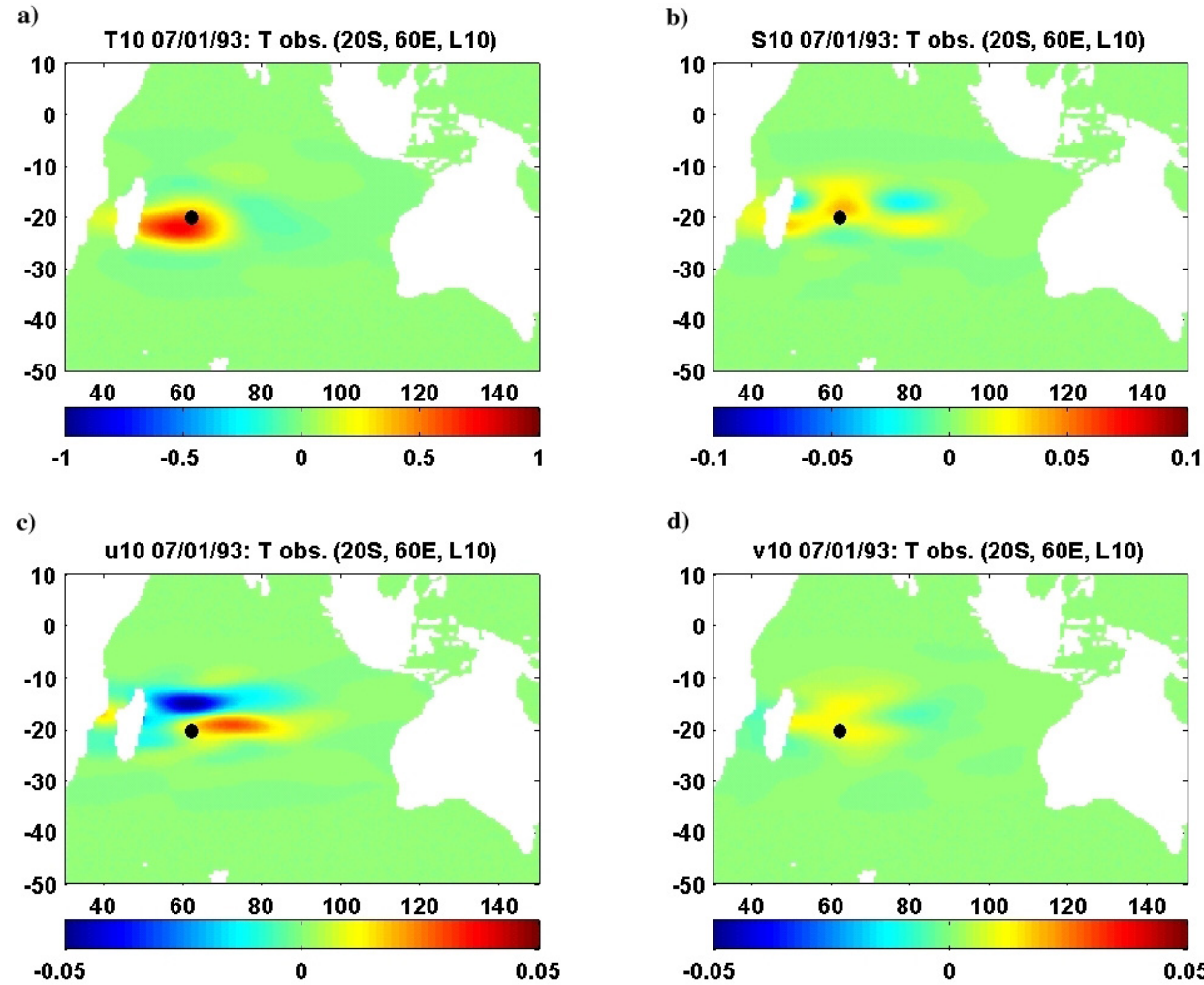

Fig. 7. Same as Fig. 6 for a hypothetical $1^{\circ} \mathrm{C}$ temperature innovation at $\left(20^{\circ} \mathrm{S}, 60^{\circ} \mathrm{E}\right)$. 

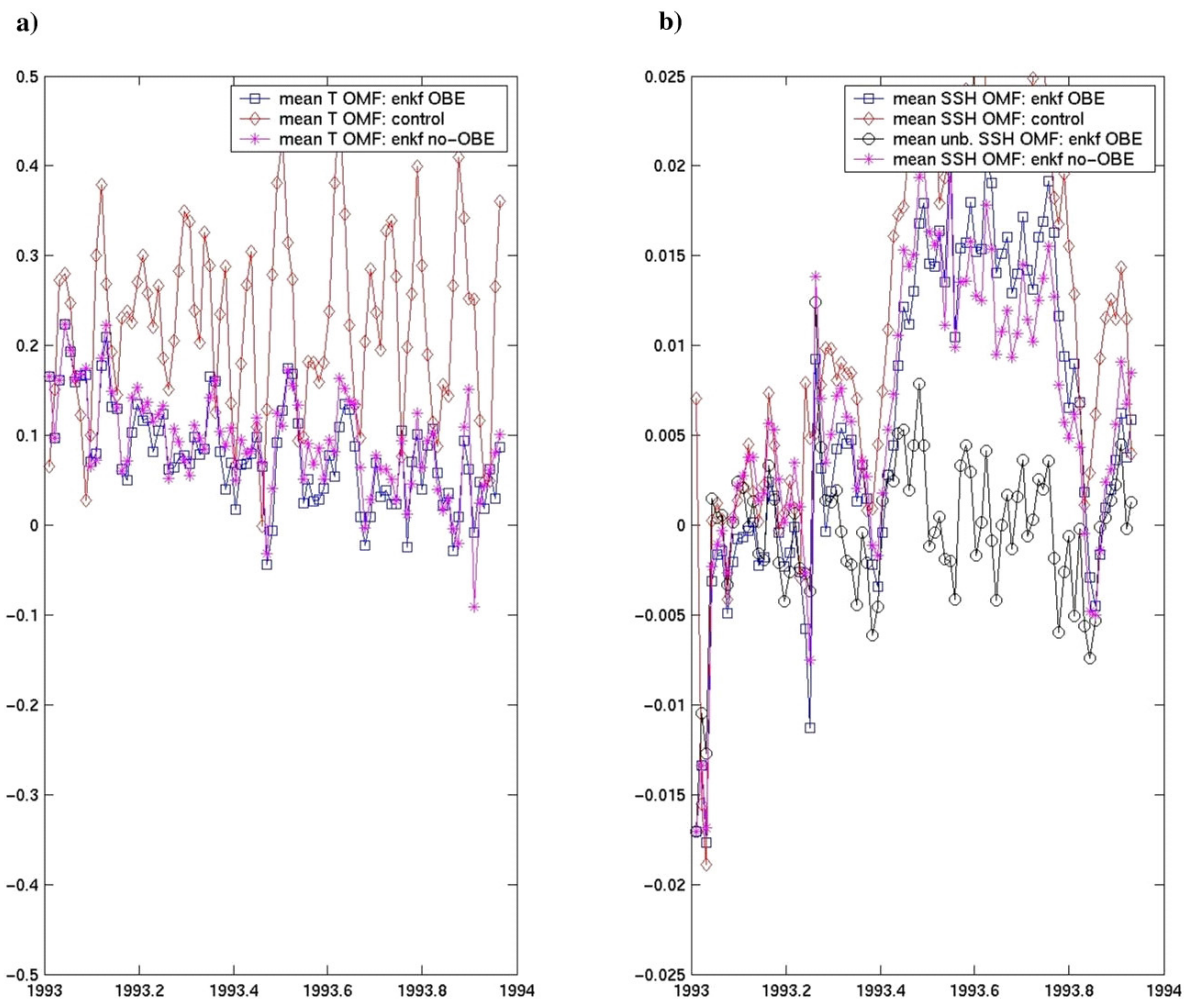

Fig. 8. Temporal evolution of the spatial-mean OMF for (a) temperature and (b) SSH in the no-assimilation control run (diamonds), the EnKF run with temperature and T/P-anomaly data assimilation and continuous correction of the SSH climatology using OBC (squares) and the EnKF run with temperature and T/P assimilation without SSH bias correction (stars). The circles in (b) correspond to the difference between the T/P observations and the unbiased state estimate of the EnKF run with OBC.

OBC estimate, the biased EnKF-OBC estimate, the EnKF run, and the control run, in that order.

Figure 9 shows the evolution of RMS OMF statistics corresponding to the spatial-mean statistics displayed in Fig. 8. In both the EnKF-OBC and EnKF runs, the RMS $\mathrm{OMF}$ for temperature drops quickly from an initial value of $1.54^{\circ} \mathrm{C}$ during the first month and stay near $1.0^{\circ} \mathrm{C}$ thereafter (Fig. 9a). Averaged over the length of the runs, the RMS temperature $\mathrm{OMF}$ are $0.94^{\circ} \mathrm{C}, 1.03^{\circ} \mathrm{C}$ and $1.49^{\circ} \mathrm{C}$, in the EnKF$\mathrm{OBC}, \mathrm{EnKF}$ and control runs. Thus, although the effect of applying $\mathrm{OBC}$ in the T/P assimilation on the spatial-mean OMF statistics for temperature was hardly noticeable, the effect on the RMS temperature OMF is more significant.

As expected, the application of OBC in the T/P assimilation has more impact on the RMS OMF statistics for SSH than on the RMS temperature OMF (Fig. 9b). Indeed, the RMS OMFs of the biased state estimate from the EnKF-OBC run are very close to those of the control, while the RMS OMFs of the unbiased estimate quickly become markedly lower. The corresponding RMS statistics from the EnKF run lie between those of the control and unbiased EnKF-OBC estimate. Averaged over the entire runs, the RMS OMFs are $0.077 \mathrm{~m}, 0.092 \mathrm{~m}, 0.087 \mathrm{~m}$ and $0.094 \mathrm{~m}$ for the unbiased EnKF-OBC estimate, biased EnKF-OBC estimate, EnKF, and control, respectively.

\subsection{Hindcast experiments}

The third experiment consists of a series of coupled-model hindcast initializations to examine the impact on the seasonal forecast skill of using the EnKF to process temperature and altimetry observations and of applying $\mathrm{OBC}$ in the altimeter data assimilation. Unlike the previous experiment in which a no-assimilation control run was used to measure the impact of the assimilation, the performance of the EnKF-based assimilation system is assessed in a comparison to the system used for the GMAO production seasonal forecasts. In the production system, only temperature data are assimilated using a standard optimal interpolation (OI) algorithm and a salinity correction is applied in an attempt to preserve the temperature and salinity properties of water masses (Troccoli et al., 2003).

In the hindcast initialization experiment, a three-month EnKF run is started on February 1 of each year from 1993 to 2003. The setup of each run is identical to that of the EnKF-OBC run of the previous experiment, i.e. temperature and T/P data are assimilated and OBC is used to continually adjust the model SSH climatology which is used to calculate forecast SSH anomalies. Following the three months of assimilation, the final state of each of the first five ensemble members is provided as an initial ocean state to an instance of the CGCM, which is then run in forecast mode without 
a)

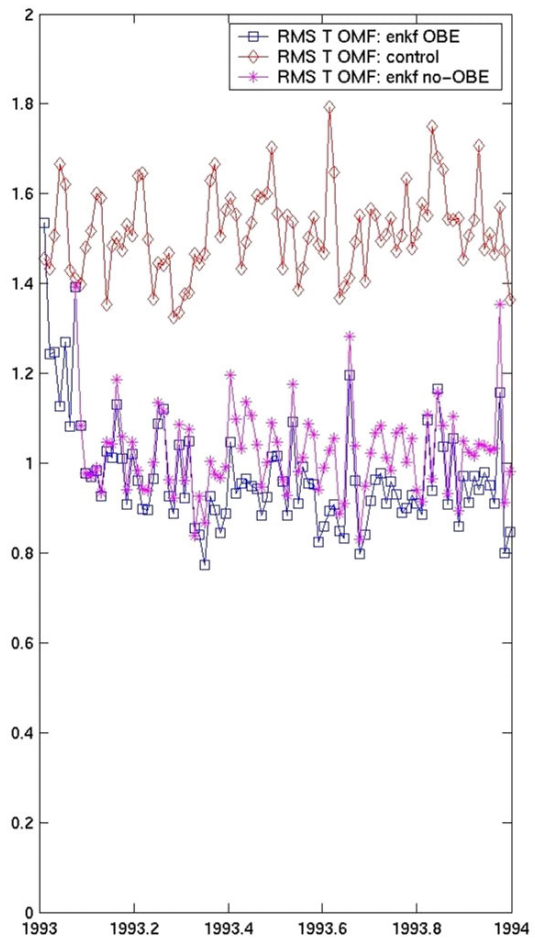

b)

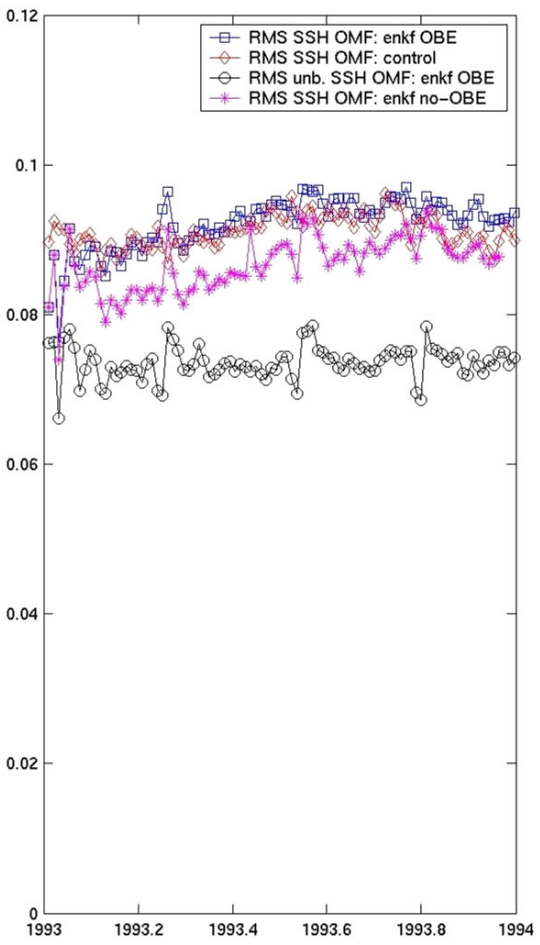

Fig. 9. Same as Fig. 8 for the spatial RMS OMF differences.

assimilation for 12 months. Only five of the 16 EnKF-OBC ensemble members are used in order to reduce the computational expense of the experiment.

The impact of data assimilation on the coupled-model seasonal-prediction skill can be assessed from a comparison of CGCM hindcasts of Niño-3.4 SST anomalies used with observed SST anomalies from the Reynolds data set (Reynolds and Smith, 1994). This is done in Fig. 10 for the production system (Fig. 10a) and for the EnKF-based system (Fig. 10b). Although the production system produces an excellent hindcast of the major 1997/1998 El Niño and correctly predicts the minor 1995 warm event, it fails to predict the 2003 El Niño and generates false La Niña alerts in 1996 and 2000. It also produces a false El Niño alert in 2001. In contrast, the system with EnKF-OBC initialization slightly underestimates the amplitude of the major 1997/1998 event and fails to predict the 1995 and 2003 events. Yet, it produces none of the false El Niño and La Niña alerts generated by the production system. There is also one ensemble member that more accurately predicts the timing of the 1997/1998 peak and the later stages of the 1998 La Niña. An examination of Niño-3 and of Niño-4 SST hindcasts confirms that the system with EnKF-OBC initialization tends to underestimate the amplitude of warm events.

The effect of the temperature+T/P data assimilation with the EnKF on Niño-3.4 SSH-anomaly hindcasts is illustrated in Fig. 11 where the SSH hindcasts obtained with the EnKFbased and production systems are shown together with a time series of observed Niño-3.4 SSH anomalies from the T/P altimeter. Much as is the case for temperature, the hindcasts using ocean initial conditions obtained from EnKFOBC runs underestimate the amplitude of the large positive SSH anomalies of nearly $20 \mathrm{~cm}$ observed at the time of the 1997/1998 El Niño. The production system also underpredicts the SSH anomalies during this major warm event, but to a lesser extent. Yet, the effect of the altimeter data assimilation with bias correction on the SSH hindcasts is generally manifested by less bias than in the hindcasts obtained with the production system. The effect on the bias is particularly noticeable in the May 1993-April 1994 and May 2000-April 2001 hindcasts.

\section{Conclusions}

The popularity of the EnKF is growing. Although a dozen or so ensemble-based approximate Kalman filters have been proposed, applications to realistic ocean or atmospheric models are few and far between. When an operational model is used and real observations are involved, issues such as how to account for model errors and bias become important. The situation is complicated by the fact that computational limitations mandate the use of small ensembles. Besides, the relative scarcity of observations of the ocean subsurface makes it difficult to verify and validate assimilation products.

In this paper, an attempt is made to overcome these challenges in an application to a high-resolution global ocean forecast model where in situ temperature observations and 
a)

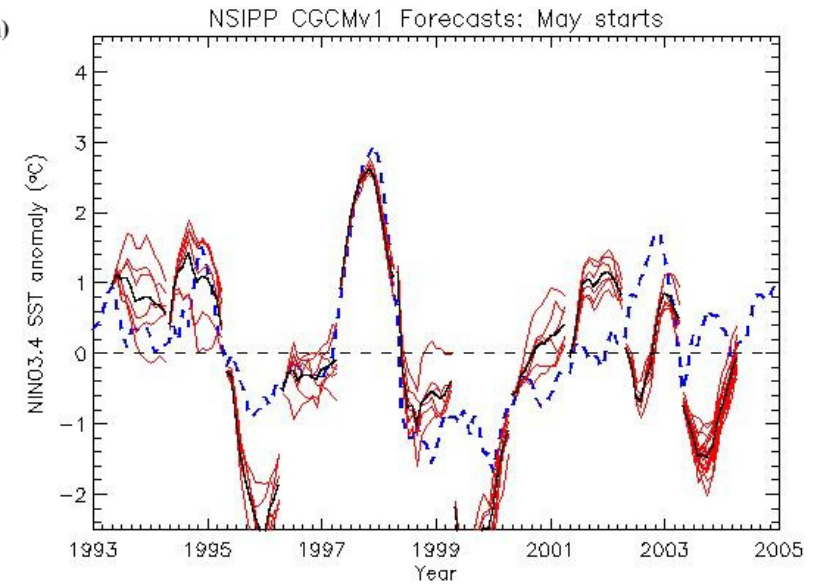

b)

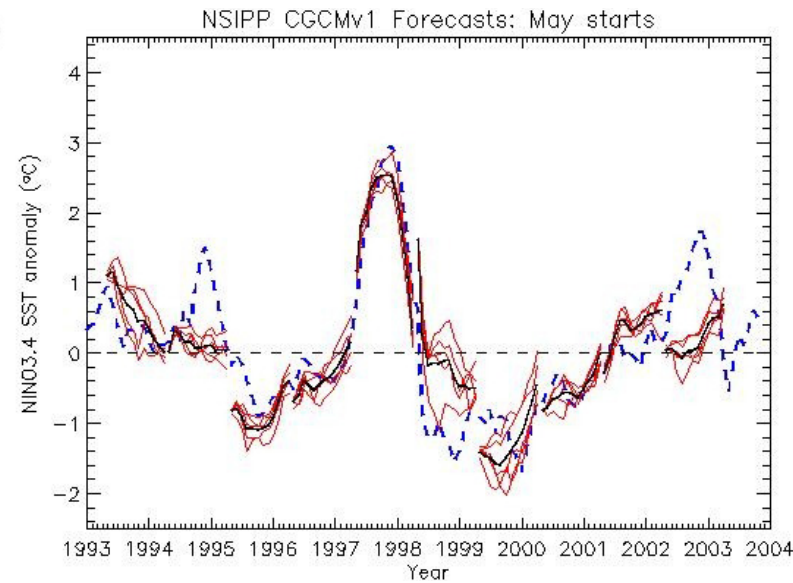

Fig. 10. Time series of Niño-3.4 SST-anomaly hindcasts.made with ensembles of the GMAO CGCM in which the ocean-model component is initialized with (a) the temperature-OI system used in production runs and (b) the EnKF system with assimilation of temperature and T/P data and use of OBC in the altimeter data assimilation. The individual ensemble members are shown in red, the ensemble mean in black. The dotted line corresponds to observed Reynolds SSTs. For computational economy, only the first five ensemble members from the EnKF-OBC runs are used in the hindcasts shown in (b). The version of the production system used in (a) is based on six-member ensembles.

satellite altimeter data are assimilated. From this perspective, the problem of forecast-model bias is exacerbated by alterations of the model SSH climatology that occur as a consequence of the assimilation of SSH anomalies. The validation and hindcast experiments described herein indicate that a simple modification of the EnKF alleviates the problem. Still, it remains to be seen whether the benefits of the adaptive bias correction methodology applied here justify its choice over simpler online and offline methodologies. Essentially, the cost of the analysis is doubled since the update equations are solved twice, first to update the bias estimate then the model state. If the same covariance localization scales were used for the bias and random error components, assuming $\boldsymbol{b}_{a}^{f}=\boldsymbol{b}_{k}^{f}$ would allow one to only solve one set of equations, but the results confirm that it is best to use larger covariance scales in the bias update.

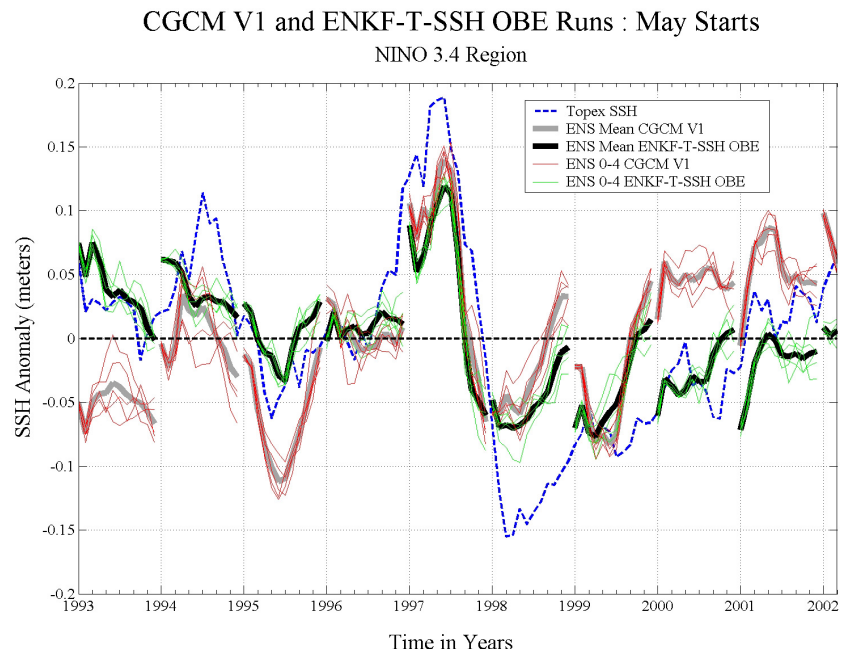

Fig. 11. Similar to each panel of Fig. 10, but for the SSH field and comparing the production seasonal forecast runs with the EnKFinitialized runs using bias correction in the altimeter data assimilation. The dotted line shows the T/P anomalies. The first five ensemble members are shown in red for the production forecasts and in green for the forecasts initialized from EnKF-OBC runs. The corresponding ensemble means are colored gray for the production system and black for the system initialized with EnKF-OBC.

Although the results suggest that the EnKF has the potential to outperform standard assimilation methodologies in seasonal forecasting applications, more validation experiments are required before definitive conclusions can be drawn. In terms of filter performance, the small ensemble size is likely the most serious limiting factor. Since halving the model grid spacing demands roughly the same increase in computational resources as quadrupling the ensemble size, a tradeoff between these two factors must always be found. Perhaps, the results obtained in the present work could be improved by running larger ensembles of a coarser-resolution version of the model.

Another question that remains unanswered is whether inflating the covariances by means of random state perturbations, as is done in this work, is generally superior to traditional multiplicative (or additive) covariance inflation. With the model used here, any level of multiplicative inflation clearly worsens OMF statistics, but the situation may be different in another context.

Acknowledgements. We thank R. Kovach for her help with the TOPEX data and Anna Borovikov for providing us with the empirical orthogonal functions of the model's variability. This work is supported by NASA's Physical Oceanography Program under RTOP 622-48-04. The computing resources were provided by the NASA Center for Computational Sciences.

Edited by: Z. Toth

Reviewed by: P. J. van Leeuwen and another referee 


\section{References}

Adamec, D.: Modulation of the seasonal signal of the Kuroshio Extension during 1994 from satellite data, J Geophys. Res.-Oceans 103, 10 209-10 222, 1998.

Anderson, J. L., and Anderson S. L.: A Monte Carlo implementation of the nonlinear filtering problem to produce ensemble assimilations and forecasts, Mon. Wea. Rev., 127, 2741-2758, 1999.

Bloom, S., Tacaks, L., DaSilva, A., and Ledvina, D.: Data assimilation using incremental analysis updates, Mon. Wea. Rev., 124, 1256-1271, 1996.

Borovikov, A. Y., Rienecker, M., and Keppenne, C. L.: Multivariate error covariance estimates by Monte-Carlo simulation for assimilation studies in the Pacific Ocean, Mon. Wea. Rev., in press, 2005.

Burgers, G., van Leeuwen, P., and Evensen, G.: Analysis scheme in the ensemble Kalman filter, Mon. Wea. Rev., 126, 1719-1724, 1998.

Carton, J. A., Chepurin, G., Cao, X., and Giese, B. S.: A Simple Ocean Data Assimilation analysis of the global upper ocean 1950-1995, Part 1: methodology, J. Phys. Oceanogr., 30, 294 $309,2000$.

Dee, D. P. and da Silva, A. M.: Data assimilation in the presence of forecast bias, Quart. J. Roy. Meteor. Soc., 124, 545, 269-295, 1998.

Dee, D. P., and Todling, R.: Data assimilation in the presence of forecast bias: the GEOS moisture analysis, Mon. Wea. Rev., 128, 545, 3268-3282, 2000.

Evensen, G.: Sequential data assimilation with a nonlinear quasigeostrophic model using Monte Carlo methods to forecast error statistics, J. Geophys. Res., C99, 10 143-10 162, 1994.

Evensen, G.: The ensemble Kalman filter: theoretical formulation and practical implementation, Ocean Dynamics, 53, 343-367, 2003.

Friedland, B.: Treatment of bias in recursive filtering, IEEE Transactions on Automatic Control, AC-14, 359-367, 1969.

Gaspari, G. and Cohn, S.: Construction of correlation functions in two and three dimensions, Quart. J. Roy. Meteor. Soc., 125, 723 757, 1999.

Houtekamer, P. and Mitchell, H.: Data assimilation using an ensemble Kalman filter technique, Mon. Wea. Rev., 126, 796-811, 1998.

Houtekamer, P. and Mitchell, H.: A sequential ensemble Kalman filter for atmospheric data assimilation, Mon. Wea. Rev., 129, 123-137, 2001.

Kalman, R.: A new approach to linear filtering and prediction problems, J. Basic Eng., D82, 35-45, 1960.
Keppenne, C. L. and Rienecker, M.: Design and Implementation of a Parallel Multivariate Ensemble Kalman Filter for the Poseidon Ocean General Circulation Model, NASA Tech. Memo2001-104606, 21., 31, 2001

Keppenne, C. L. and Rienecker, M.: Development and Initial Testing of a Parallel Ensemble Kalman Filter for the Poseidon Isopycnal Ocean General Circulation Model, Mon. Wea. Rev., 130, 2951-2965, 2002.

Keppenne, C. L. and Rienecker, M.: Assimilation of temperature into an isopycnal ocean general circulation model using a parallel ensemble Kalman filter, J. Mar. Sys., 40-41, 363-380, 2003.

Konchady, M., Sood, A., and Schopf, P.: Implementation and performance evaluation of a parallel ocean model, Parallel Comput., 24, 181-203, 1998.

Martin, M. J., Bell, M. J., and Nichols, N. K.: Estimation of systematic error in an equatorial ocean model using data assimilation, Int. J. Numer. Meth. Fluids, 40, 435-444, 2002.

Pacanowski, R. and Philander, S.: Parameterization of vertical mixing in numerical models of the tropical oceans, J. Phys. Oceanogr., 11, 1443-1451, 1981.

Reynolds, R., and Smith, T.: Improved global sea-surface temperature analyses using optimum interpolation, J. Climate, 7, 929948, 1994.

Schopf, P. and Loughe, A.: A reduced-gravity isopycnic ocean model-hindcasts of El Niño, Mon. Wea. Rev., 123, 2839-2863, 1995.

Tippett, M. K., Anderson, J. L., Bishop, C. H., Hamill, T. M., and Whitaker, J. S.: Ensemble square root filters, Mon. Wea. Rev., 131, 1485-1490, 2003.

Sterl, A. and Kattenberg, A.: Embedding a mixed layer model into an ocean general circulation model of the Atlantic: The importance of surface mixing for heat flux and temperature, J. Geophys. Res., 99, 14 139-14 157, 1994.

Troccoli, A., Rienecker, M. M., Keppenne, C. L., and Johnson, G. C.: Temperature data assimilation with salinity corrections: validations for the NSIPP ocean data assimilation system in the Tropical Pacific ocean, 1993-1998, NASA Tech. Memo-2003104606, 24, 23, 2003.

van Leeuwen, P. J.: An ensemble smoother with error estimates, Mon. Wea. Rev., 129, 709-728, 2001.

van Leeuwen, P. J.: A variance-minimizing filter for large scale applications, Mon. Wea. Rev., 131, 2071-2084, 2003.

Vossepoel, F. C., Weaver, A. T., Vialard, J., and Delecluse, P.: Adjustment of near-equatorial wind stress with four-dimensional variational data assimilation in a model of the Pacific Ocean, Mon. Wea. Rev., 132, 2070-2083, 2004.

Yang, S., Lau, K., and Schopf, P.: Sensitivity of the tropical Pacific Ocean to precipitation induced freshwater flux, Clim. Dynam., $15,737-750,1999$. 